\title{
Sentiment d'efficacité personnelle en gestion des comportements. Élaboration et validation d'une échelle de mesure
}

\section{Malika S. Dessibourg}

Les études concernant le sentiment d'efficacité personnelle et sa mesure montrent qu'il ne s'agit pas d'une caractéristique globale mais d'un ensemble de convictions que peut avoir un individu à propos de différents aspects de son fonctionnement. Cet article présente la construction et les premiers éléments de validation de l'échelle de mesure du sentiment d'efficacité personnelle propre à la gestion des comportements en classe au secondaire I. Elle a été élaborée et validée selon les recommandations de Bandura (2006) auprès d'une population d'enseignants en deux étapes : une phase pilote suivie d'une phase de test. La validité de contenu a été vérifiée à l'aide d'une analyse conceptuelle approfondie et d'un accord inter-juges. La validité de construit a été vérifiée à l'aide d'une analyse en composante principale lors de la phase pilote puis d'une analyse factorielle confirmatoire lors de la phase test. Ces analyses soulèvent des questions en ce qui concerne la validation du modèle utilisé. La version finale comporte 16 items et satisfait les conditions de fiabilité.

\section{Introduction}

Cette recherche s'articule autour de la construction et de premiers éléments de validation d'une échelle mesurant le sentiment d'efficacité personnelle touchant à un aspect spécifique de l'éducation en milieu scolaire: la gestion des comportements en classe de l'enseignant ${ }^{1}$ ordinaire, pour une classe ordinaire du secondaire $\mathrm{I}^{2}$. Celle-ci est destinée à être utilisée dans le cadre de la formation initiale des enseignants et pourrait également servir à soutenir et former les enseignants confirmés dans le cadre de formations continues ou d'auto-évaluations, par exemple. Cet article est développé de manière à aborder les étapes importantes de la construction d'une échelle en fonction des recommandations de Bandura (2006). Dans un premier temps, la problématique est expliquée. Ensuite, le contexte de la recherche ainsi que le cadre conceptuel, essentiels à la compréhension des choix effectués concernant les items de l'échelle, seront développés. Enfin, la méthodologie et les premiers éléments de validation seront exposés. L'article se terminera par la discussion et la conclusion. 


\section{Problémat ique}

La gestion des comportements en classe est très souvent citée comme l'un des plus grands défis auxquels doivent faire face les enseignants du secondaire I, débutants ou non (Dicke, Elling, Schmeck \& Leutner, 2015; Leveillé \& Dufour, 1999; Sempowicz \& Hudson, 2011). La littérature montre qu'un des éléments importants pouvant porter une influence sur la qualité de la gestion des comportements en classe est le sentiment d'efficacité personnelle des enseignants (Gaudreau, Royer, Beaumont \& Frenette, 2012). Le sentiment d'efficacité personnelle peut être amélioré de manière significative durant la formation initiale des enseignants et ce même dans des cas où les enseignants en formation ont déjà un sentiment d'efficacité personnelle plutôt élevé (Brown, Lee \& Collins, 2015). Toutefois, il semble y avoir un manque au niveau de la formation car la gestion des comportements en classe reste un défi important pour les jeunes enseignants (Eisenman, Edwards \& Cushman, 2015; Hamilton, 2015). Il serait donc pertinent de mettre en place un système de formation à la gestion des comportements comprenant des outils permettant de prendre en compte les besoins des étudiants en difficulté tout en agissant sur leur sentiment d'efficacité personnelle. La construction de l'échelle présentée dans cette recherche s'inscrit dans cette démarche.

Les différences existant d'un individu à un autre concernant le sentiment d'efficacité personnelle, son développement et son intégration sont reconnues par la théorie de l'efficacité personnelle. Ce sentiment n'est pas considéré comme une caractéristique globale, mais comme «un ensemble différencié de croyances sur soi liées à des domaines distincts de fonctionnement [...] les croyances d'efficacité se différencient à l'intérieur même de ces domaines d'activité» (Bandura, 2013, p. 62). Ceci explique la nécessité de cibler et contextualiser les échelles de mesure du sentiment d'efficacité personnelle dans différents domaines (Bandura, 2006). En plus de l'opérationnalisation que Bandura (2013) a fait de sa propre théorie dans la création d'une échelle de mesure du sentiment d'efficacité des enseignants, plusieurs échelles mesurant le sentiment d'efficacité personnelle de l'enseignant basées sur cette dernière ont été élaborées (Dembo \& Gibson, 1985; Schwarzer, Schmitz \& Daytner, 1999; Tschannen-Moran \& Woolfolk Hoy, 2001). Jusqu'à maintenant, ces échelles s'intéressaient à l'acte d'enseigner dans son entier (Bandura, 2013; Schwarzer et al., 1999; Tschannen-Moran \& Woolfolk Hoy, 2001) ce qui présente déjà une forme de contextualisation, bien que plusieurs chercheurs aient émis des doutes quant au fait que le sentiment d'efficacité personnelle des enseignants puisse être mesuré comme un construit unique (Deemer \& Minke, 1999; Duffin, French \& Patrick, 2012; Henson, 2001; Tschannen-Moran \& Woolfolk Hoy, 2001). Plus récemment toutefois, l'échelle du sentiment d'efficacité personnelle des enseignants en gestion de classe et comportant de nombreux aspects de la gestion des comportements a été développée par Gaudreau, Frenette et Thibodeau (2015), s'intéressant ainsi à un aspect plus précis du sentiment d'efficacité personnelle des enseignants. 
Malgré cela, il n'existe que peu d'outils réellement ciblés sur ce sujet (Abu-Tineh, Khasawneh \& Khalaileh, 2011; Gaudreau et al., 2015). De plus, à notre connaissance, il n'existe à l'heure actuelle, aucun outil mesurant le sentiment d'efficacité personnelle de l'enseignant spécifique à la gestion des comportements au secondaire I. Or, d'après Bandura (2006), pour qu'une échelle de mesure du sentiment d'efficacité personnelle soit le plus efficace possible, elle doit se centrer sur des aspects ciblés plutôt que généraux. Allant dans ce sens, plusieurs chercheurs s'intéressant au sentiment d'efficacité personnelle des enseignants s'accordent sur le fait que l'enseignement est complexe et que les mesures le concernant devraient prendre en compte ses différentes facettes (Duffin et al., 2012; Tschannen-Moran \& Woolfolk-Hoy, 2001; Tschannen-Moran, Woolfolk Hoy \& Hoy, 1998). À cela, ajoutons que la recherche dans le domaine du sentiment d'efficacité personnelle des enseignants est quelque peu confuse, surtout en ce qui concerne sa mesure (Henson, 2001). Il semble de ce fait pertinent d'élaborer une échelle spécifique à un aspect de l'enseignement répondant à un besoin de la formation actuelle, tout en prenant en compte les difficultés rencontrées par le passé dans la mesure du sentiment d'efficacité personnelle.

\section{Contexte de la recherche: Le secondaire I}

\section{Les élèves et l'école}

Les élèves quittent le degré primaire et arrivent au secondaire I vers 12 ans, durant la période de passage du stade des opérations concrètes au stade des opérations formelles appelé aussi stade hypothético-déductif selon Piaget (Piaget \& Inhelder, 1966). C'est à ce moment qu'ils passent également par de nombreuses modifications physiques. Ce stade est caractérisé par la capacité d'abstraire son raisonnement des objets et de l'environnement qui l'entourent: ils peuvent donc non seulement créer des hypothèses mais aussi utiliser les événements du passé pour développer leurs croyances et leurs idées, ayant ainsi accès à de nouvelles structures du raisonnement (Piaget $\&$ Inhelder, 1966). Cette capacité acquise de pouvoir raisonner sur le possible et l'impossible, le réel et l'abstrait, pousse l'adolescent à tester ses limites et à observer les réactions des personnes qui l'entourent face aux points de vues souvent invraisemblables qu'il soutiendra, adoptant ainsi un mécanisme de défense que Freud appelle intellectualisation (Freud, 1978). A ce moment, l'adolescent a principalement besoin de justification logique face aux nombreuses discussions animées ou conflits dans lesquels il peut se lancer avec des personnes adultes (Freud, 1978). Le passage d'un stade à l'autre ne se fait pas du jour au lendemain. Ainsi, l'adolescent va passer par une phase de préparation du stade formel durant laquelle il va traverser une période de mélanges des raisonnements et des logiques, se comportant quelques fois de façon mature et d'autres fois non (Piaget \& Inhelder, 1966). Ce moment, auquel on fait parfois informellement référence en parlant de crise d'adolescence est en fait ce qu'Erikson 
(1972) qualifie de crise d'identité. Cet état transitoire de fort déséquilibre se résout normalement lors de l'accès au stade formel (Wagner, 1987).

Par ailleurs, cette recherche est basée dans un contexte dans lequel le passage de l'école primaire au secondaire I représente également de nombreux changements pour les adolescents concernés: les élèves sont amenés à changer d'établissement scolaire, les classes sont dissoutes puis reformées en fonction de leurs capacités scolaires, amenant les élèves à se retrouver avec de nouvelles personnes, souvent inconnues. Le fonctionnement de l'école du secondaire I est très différent de celui de l'école primaire: il n'y a plus un à deux enseignants par classe, mais autant d'enseignants que de branches enseignées, avec parfois un enseignant pour plusieurs branches. Ainsi, selon les écoles, les élèves ou les enseignants sont amenés à changer de classe plusieurs fois par jour.

\section{Les parents}

Au secondaire I, plus de responsabilités sont données à l'élève et le lien entre l'école et les parents devient parfois moins présent. Or, plusieurs recherches ont mis en lien l'implication des parents avec la réussite scolaire et éducative ainsi qu'une meilleure attitude vis-à-vis de l'école de la part des élèves, entre autres effets positifs (Blaya, Gilles, Plunus \& Tièche, 2011; Gonzalez-DeHass, Willems \& Doan Holbein, 2005). Pour un enseignant, établir une relation de confiance avec les parents permet de favoriser la communication entre les parents et l'école dans un premier temps (Adams \& Christenson, 2000), mais permet également aux parents de reconnaître l'expertise et la compétence de l'école et de ses intervenants, marquant ainsi les limites à leur implication et faisant d'eux un partenaire qui participe aux décisions (De Saedeleer, Brassard \& Brunet, 2004). Ce dernier aspect semble particulièrement important concernant la gestion du comportement de leur enfant. Enfin, les enseignants ayant un sentiment d'efficacité élevé ont généralement plus de facilité à inclure les parents dans la vie scolaire de leurs enfants (Dembo \& Gibson, 1985; Hoover-Dempsey, Walker, Jones \& Reed, 2002). Cet aspect a donc été pris en compte pour l'élaboration de l'échelle de mesure du sentiment d'efficacité personnelle présentée dans cette étude.

\section{Synthèse}

Ces éléments en lien avec les élèves, le contexte dans lequel ils évoluent et la relation parents-école nous mènent à penser que la gestion des comportements durant cette période doit se faire selon des stratégies différentes de celles appliquées à d'autres moments de la scolarité obligatoire et post-obligatoire. La littérature vient d'ailleurs soutenir cette idée que la gestion des comportements est un sujet particulièrement important au niveau du secondaire I: en plus d'être une période durant laquelle une tendance élevée d'actes de rebelle peut être observée, de nombreuses stratégies utilisées à l'école primaire perdent de leur efficacité avec les élèves plus âgés du secondaire (Malmgren, Trezek \& Paul, 
2005). Notons pour terminer que les enseignants en formation du secondaire I présentent en général un sentiment d'efficacité personnelle concernant la gestion de classe plus faible que celui de leur collègues du primaire (Christophersen, Elstad, Turmo \& Solhaug, 2016).

\section{Cadre conceptuel}

\section{La gestion de classe}

Il existe différentes définitions de la gestion de classe. Cette recherche aborde la gestion de classe comme un terme générique évoluant avec son temps et désignant tout ce que peut faire un enseignant au sein de sa classe afin de mener à bien la planification ainsi que l'organisation de toutes les situations d'enseignement-apprentissage auxquelles il fera face dans l'exercice de ses fonctions (Nault \& Fijalkow, 1999). Cette définition plutôt générale a l'avantage de mettre en évidence l'aspect évolutif du concept et de l'enseignant. De plus, par rapport à cette définition de la gestion de classe, la gestion des comportements n'en est qu'un aspect. Il semble important de le souligner car cette recherche s'intéresse seulement à la gestion directe des comportements, c'est-à-dire aux actions qui ont comme premier but de gérer les comportements, sans pour autant stipuler qu'il s'agisse de l'aspect principal ou unique de la gestion de classe. Plusieurs chercheurs abondent dans ce sens, bien que leurs définitions varient quelque peu les unes des autres (Doyle, 2006; Gaudreau et al., 2012; Martineau \& Gauthier, 1999).

\section{La gestion des comportements et quelques modèles existants}

La gestion des comportements fait partie intégrante de l'enseignement: elle favorise l'apprentissage des élèves (Martineau \& Gauthier, 1999) et elle représente une forme d'aide apportée aux élèves dans la capacité d'organiser leur travail, leur matériel et leurs apprentissages (Sieber, 2000). De plus, elle permet l'apprentissage de règles sociales sans lesquelles l'apprentissage des connaissances et des savoirs scolaires n'est pas possible (Sieber, 2000). L'indiscipline d'une classe n'étant pas une donnée constante, la gestion des comportements demande à l'enseignant qu'il soit capable de s'adapter d'année en année (Nault \& Fijalkow, 1999).

Il existe plusieurs modèles reconnus de la gestion des comportements en classe s'inscrivant dans des approches différentes qui peuvent être qualifiés d'interventionniste, interactionniste ou non-interventionniste (Charles, 2009; Glickman \& Tamashiro, 1980; Legault, 2001). Les approches interventionnistes sont celles dans lesquelles l'enseignant fait preuve de plus de contrôle sur l'élève qui est perçu comme un être ayant besoin d'être conditionné et contrôlé (Glickman \& Tamashiro, 1980). Kounin (1970) qui prône la gestion préventive 
par l'instauration d'un rythme de travail soutenu et par la vigilance de l'enseignant sachant intervenir rapidement et au bon moment est un exemple parlant de cette approche. À l'opposé, se trouvent les approches non-interventionnistes: celles où l'élève bénéficie de plus d'autonomie (Glickman \& Tamashiro, 1980), comme par exemple dans le modèle de Gordon (Charles, 2009; Gordon, 2009) qui prône l'autodiscipline des élèves en prenant les décisions relatives à la classe conjointement avec eux, en écoutant passivement un élève qui en aurait besoin ou encore en évitant les obstacles à la communication tels que: commander, conseiller ou complimenter pour n'en citer que quelques-uns. À mi-chemin entre les deux, nous retrouvons les modèles interactionnistes dans lesquelles l'élève est perçu comme un individu pouvant se contrôler mais à l'intérieur de certaines limites (Glickman \& Tamashiro, 1980; Legault, 2001). Un exemple de modèle s'inscrivant dans cette approche est celui de Dreikurs (1968) dans lequel l'enseignant cherche à neutraliser ce qu'il appelle les «buts erronés», à savoir tout autre objectif poursuivi par l'élève qui ne parvient pas à atteindre le but fondamental d'appartenance, afin de se sentir important. Ce modèle encourage les enseignants à faire cela sur un ton amical plutôt que par la punition afin d'amener l'élève à examiner son comportement. L'élève est ainsi responsabilisé et appelé à respecter les autres ainsi que soi-même (Charles, 2009).

\section{Difficulté d'application des modèles existants dans la pratique}

La plupart du temps, il est très difficile, voire impossible pour un enseignant de se référer entièrement à un modèle de gestion des comportements. Il existe plusieurs raisons expliquant cela: ces modèles peuvent être très théoriques, et de ce fait difficilement applicables à la classe. Même les modèles plus pratiques comme celui de Canter (2010) peuvent présenter des difficultés d'adaptation pour les enseignants. Cela peut être expliqué par le fait que la personnalité ainsi que les croyances de l'enseignant sont pour beaucoup dans les choix faits concernant la gestion des comportements. Chaque enseignant devrait donc prendre en compte ses besoins et ce qu'il apprécie ou non dans sa classe (Charles 2009; Legault, 2001). À cela, ajoutons le fait que tous les problèmes survenant en classe ne se règlent pas de la même façon: tout comme chaque enseignant a des particularités dues à son caractère, il existe chez les élèves plusieurs types de profils comportementaux (Sieber, 2000). Chaque situation représente donc un cas différent selon le contexte et le caractère des individus en jeu. De ce fait, nous postulons qu'un même enseignant peut s'inscrire dans une approche interventionniste, interactionniste ou non-interventionniste et ce dans un même cours.

\section{Modèle de gestion des comportements au secondaire I}

La gestion des comportements doit favoriser les apprentissages mais aussi le bien-être des élèves, sans quoi des effets pervers liés à une gestion inefficace ou nuisible risquent de mettre en péril le bon fonctionnement de la classe ainsi que la 
réussite scolaire des élèves (Martineau \& Gauthier, 1999). Il n'existe pas qu'une façon de mettre en place un système de gestion des comportements: celui-ci doit être adapté à la personne qui l'applique ainsi qu'à son public. Afin de mieux appréhender les situations ainsi que les choix auxquels font face les enseignants par rapport au comportement à adopter, les différentes composantes de la gestion des comportements d'élèves vont être présentées. Elles sont modélisées en quatre phases et quatre dimensions de la gestion des comportements directe, c'est-à-dire avec pour objectif principal d'agir sur les comportements. Cette modélisation a été réfléchie à partir des modèles synthétisés par Charles (2009) ainsi que celui, plus récent, de Sieber (2000).

\section{Quatre phases}

Pour les besoins de la présente recherche, tous les comportements que peuvent adopter les enseignants dans le but de favoriser la gestion des comportements selon les modèles existants les plus reconnus, synthétisés par Charles (2009), ont été répertoriés et triés. Ils ont ensuite été catégorisés. Ce travail permet une vision d'ensemble des choix possibles pour un enseignant en ce qui concerne la gestion des comportements (annexe 1). En faisant ce tri, il semblait qu'une phase manquait: la remédiation, qui intervient lorsqu'une correction a déjà eu lieu et qui demande un effort supplémentaire de la part de l'enseignant dans le but de trouver de nouvelles solutions à un problème de comportement. Ainsi, ce n'est pas trois phases comme suggérées par Charles (2009) qui ont été définies mais quatre: la prévention, le soutien, la correction et la remédiation. Elles sont définies comme suit:

- La prévention: elle se rapporte à tout ce que l'enseignant peut faire ou dire à l'avance pour prévenir les comportements indésirables au sein d'une classe. Elle est normalement mise en place dès le début de l'année scolaire. Par exemple: expliciter les attentes en lien avec les comportements attendus.

- Le soutien: il se réfere à tout ce que peut faire l'enseignant pour encourager et inciter les comportements positifs à ses élèves sur la durée. Comme la prévention, le soutien est normalement mis en place dès le début de l'année scolaire. Par exemple: expliquer aux élèves que l'on apprécie leur bon comportement.

- La correction: elle est tout ce que peut faire un enseignant pour signaler à un élève que son comportement n'est pas adapté et qu'il doit le modifier. Cela va du simple regard appuyé à des conséquences plus importantes en fonction de la gravité du comportement adopté par l'élève.

- La remédiation: les enseignants ont recours à la remédiation lors d'écarts de conduite jugés par l'enseignant comme étant plus graves ou lors de récidive. Elle se réfere à tout ce que peut faire l'enseignant pour éviter qu'un comportement indésirable subsiste ou se reproduise. Par exemple: différencier le système de gestion des comportements pour un élève ayant déjà rencontré des problèmes de comportement. 
Ces définitions ont été retravaillées et different quelque peu des originales (Charles, 2009) afin de permettre la classification des comportements de l'enseignant de manière plus aisée. La définition qui posait problème en particulier était celle du soutien qui chevauchait parfois la prévention comme la correction. Par exemple, la confiscation d'objets, ou l'utilisation de la proximité physique pour calmer un élève qui perturbe ne sont plus considérés comme des mesures de soutien mais comme des mesures correctives. En limitant le soutien à tout ce qu'un enseignant peut faire pour encourager les bons comportements et en élargissant la correction à toute action, grande ou petite que peut faire un enseignant pour signifier à un élève que son comportement n'est pas acceptable, les zones peu claires entre ces différentes phases ont été supprimées.

\section{Quatre dimensions}

La catégorisation des comportements que peut adopter un enseignant peut ensuite s'inscrire dans quatre dimensions de la gestion des comportements: (1) gestion proactive, (2) gestion réactive, (3) implication proactive des parents ou de la personne responsable de l'élève (4) implication réactive de personnes externes à la classe.

Pour mieux comprendre le fonctionnement de la gestion des comportements, le modèle de Sieber (2000) fondé sur l'observation de nombreuses situations en contexte a été retenu. Ce modèle permet de définir trois formes de gestion des comportements. La première est la gestion organisationnelle. Cette forme de gestion est plus indirecte et concerne de ce fait la gestion de classe dans son intégralité, étant donné qu'il s'agit de la planification de l'enseignement et de l'organisation de l'espace de la salle de classe. Elle ne sera donc pas retenue dans cette recherche, axée uniquement sur la gestion directe des comportements. La seconde est la gestion préventive qui englobe tout ce que l'enseignant peut faire pour établir et rappeler le cadre pédagogique et éducatif aux élèves (Sieber, 2000). Nous avons gardé cette dimension que nous avons choisi de renommer gestion proactive pour ne pas confondre cette dimension avec l'une des phases de la gestion des comportements: la prévention. Le but de la gestion proactive est avant tout de mettre en place des dispositifs et des habitudes de classe visant à prévenir l'apparition de comportements indisciplinés, par l'élaboration de règles de classe ou des consignes claires et réfléchies en ce qui concerne le comportement à adopter, par exemple. Bien que cette dimension concerne principalement les phases de soutien et de prévention, un enseignant peut se montrer proactif lors de la phase de correction, par exemple, en incluant l'élève dans la recherche de solutions face à un comportement déplacé ou lors de la phase de remédiation, en mettant sur pied des mesures permettant d'appréhender de nouveaux écarts de conduites. Enfin, la troisième est la gestion réactive que nous avons également gardée et qui fait référence, comme son nom l'indique, à la réaction directe de l'enseignant suite aux comportements indisciplinés des élèves qui sera par exemple une réprimande. La gestion réactive peut également 
survenir à la suite d'un comportement positif qui mérite un encouragement, un feed-back positif ou des félicitations spontanées (Sieber, 2000). Dans ce cas, la gestion réactive n'intervient pas durant la phase de correction mais dans la phase de soutien. Notons que ces deux dimensions ne sont pas en opposition mais complémentaires et doivent être adaptées en fonction du contexte: l'enseignant, la classe et la situation disciplinaire.

En plus de ces deux dimensions, il paraissait cohérent de différencier la gestion faite par l'enseignant seul et la gestion faite en collaboration avec des adultes externes à la classe. Il s'agit là de contacter les parents (par écrit, par téléphone ou à l'aide d'une réunion) dans le but de les informer du fonctionnement du système de gestion des comportements et de les inclure en les informant régulièrement de la situation de leur enfant en lien avec cet aspect et, lorsque cela est nécessaire, en prenant des décisions conjointement avec eux. On peut donc parler d'implication proactive des parents, nom donné à la troisième dimension. Enfin, l'implication de personnes externes à la classe dans un but de remédiation peut également être prise en compte. Il s'agit là des personnes ressources pour l'enseignant mais également des parents lorsqu'une situation est difficile à gérer, soit parce qu'il s'agit de récidive, soit parce qu'il s'agit d'un cas plus grave ou plus difficile à gérer seul. Sont groupés sous ce terme de personnes ressources: la direction, les médiateurs ainsi que le psychologue scolaire. Cette quatrième dimension est donc appelée implication réactive de personnes externes à la classe.

\section{Sentiment d'efficacité personnelle (SEP)}

Le sentiment d'efficacité personnelle est un sentiment actuel concernant le futur: il définit ce qu'une personne se sent capable de faire dans une situation particulière qui pourrait se présenter (Bandura, 1977). Il concerne l'impression qu'a un individu d'être capable d'accomplir une certaine tâche sans comparaison avec d'autres personnes: la question est ainsi de savoir si l'individu se sent capable ou non de faire quelque chose, sans se demander si les autres le sont ou pas (Woolfolk Hoy, 2004). Personne ne peut maitriser la totalité des activités humaines et chaque individu differe des autres concernant les secteurs mais également les niveaux d'efficacité: un individu peut acquérir une certaine compétence grâce à des dons naturels, des expériences socioculturelles et des circonstances fortuites qui mèneront à des modifications des trajectoires développementales (Bandura, 1986).

\section{Sources du sentiment d'efficacité personnelle}

Plusieurs éléments peuvent influencer le sentiment d'efficacité: les états physiologiques d'un individu, l'intégration des informations concernant l'efficacité (cela varie d'une personne à l'autre), la persuasion verbale, l'expérience vicariante, c'est-à-dire l'occasion de pouvoir observer quelqu'un similaire à soi vivre une réussite dans un certain domaine, et l'expérience de maîtrise (Bandura, 2013). L'expérience de maîtrise est considérée comme étant l'influence la plus impor- 
tante étant donné qu'elle procure un retour immédiat. Toutefois, comme chaque feedback suite à l'une de ces expériences doit être traité avant d'être intégré par un individu, tous les succès ne renforcent pas forcément le sentiment d'efficacité personnelle (Henson, 2001).

\section{Le sentiment d'efficacité personnelle des enseignants}

Bien que peu de recherches se soient intéressées à l'impact réel de ces sources sur le sentiment d'efficacité personnelle des enseignants spécifiquement, une étude a mis en avant le fait que des enseignants en formation indiquent l'expérience de maîtrise comme étant ce qui les prépare le mieux à l'enseignement et que celle-ci a effectivement une influence sur leur sentiment d'efficacité personnelle (Brown et al., 2015). Tschannen-Moran et al. (1998) incluent ces sources d'informations dans l'élaboration de leur modèle théorique concernant le sentiment d'efficacité personnelle des enseignants, apportant ainsi une meilleure compréhension du phénomène.

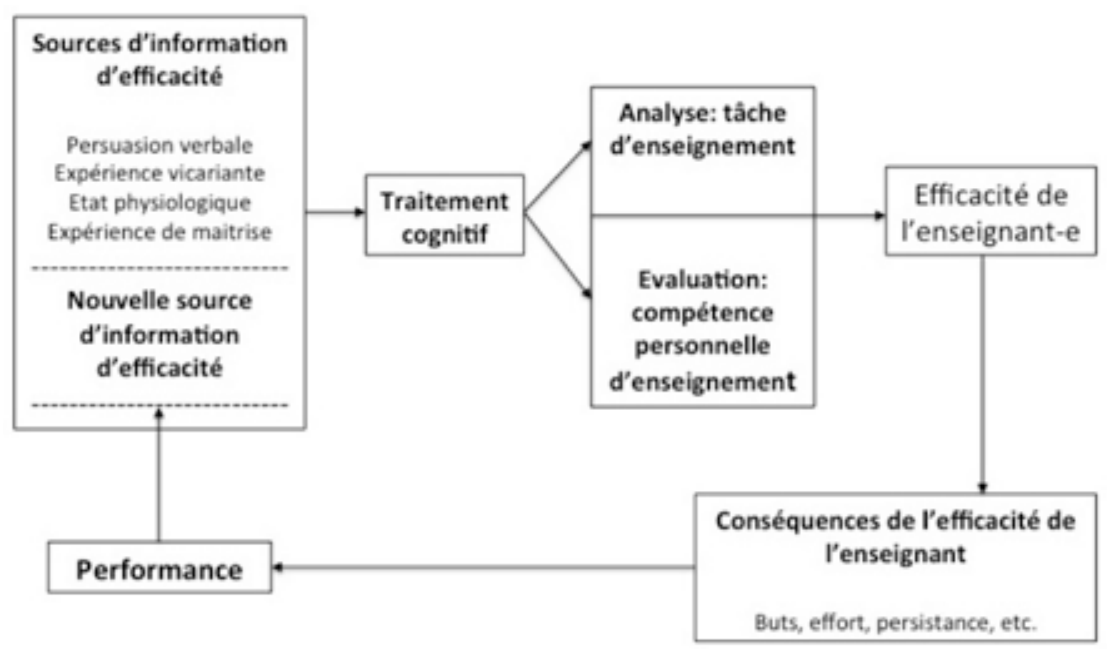

Figure 1: Modèle multidimensionnel du sentiment d'efficacité personnelle des enseignants. Tschannen-Moran et al. (1998, p. 228). Traduction libre.

Le sentiment d'efficacité personnelle joue un rôle important chez les enseignants. Celui-ci influence la motivation au travers des choix faits par un individu: un sentiment d'efficacité personnelle élevé permet à une personne de fournir plus d'efforts dans les tâches qu'elle entreprend et l'aide à faire preuve de plus de persistance face à des situations difficiles (Bandura, 2013; Woolfolk Hoy, 2004), qualités requises pour gérer les comportements au sein d'une classe. Le sentiment d'efficacité personnelle influencerait même la perception que peut avoir un enseignant de différentes tâches: un enseignant avec un fort sentiment 
d'efficacité personnelle aura tendance à percevoir une situation difficile comme un défi alors qu'un enseignant avec un sentiment d'efficacité plus faible percevra la même situation comme un obstacle (Brown et al., 2015). De par sa nature explicitement autoréférente, ce sentiment est également un bon prédicteur de comportements (Bandura, 2013). Cela nous mène donc à penser, tout comme plusieurs études l'ont confirmé par le passé (Duffin et al., 2012; Ritchie, 2006; Woolfolk Hoy, 2004), que plus le sentiment d'efficacité personnelle de l'enseignant est élevé concernant une certaine tâche, plus il sera performant. Deux individus ayant les mêmes capacités réelles à la base pourront donc obtenir des résultats différents selon leur sentiment d'efficacité personnelle. En ce qui concerne la gestion des comportements, «un enseignant qui, à un moment, doute de l'efficacité de sa façon de gérer la discipline agira différemment qu'à un autre moment où il a confiance en lui [...] Bien souvent, ce doute l'entraînera dans une spirale qui ne fera que confirmer son sentiment.» (Sieber, 2000, p. 76). En général, lorsqu'un enseignant a de faibles croyances en ce qui concerne son efficacité en lien avec la gestion des comportements, les élèves auront plus tendance à adopter des comportements difficiles en classe. En outre, plus ces comportements sont nombreux, plus l'enseignant doutera de ses capacités. Cela aura pour conséquences l'épuisement professionnel et une vulnérabilité plus élevée au stress (Gaudreau et al., 2012).

Le sentiment d'efficacité personnelle de l'enseignant a également un impact sur ses élèves: il va non seulement influencer positivement leur réussite scolaire mais également leur motivation (Duffin et al., 2012). De plus, il a été démontré que ce sentiment influe aussi sur la perception qu'ont les enseignants d'élèves ayant des difficultés comportementales (Gaudreau et al., 2012). De manière générale, les élèves d'enseignants ayant un sentiment d'efficacité élevé réussissent mieux que les autres (Henson, 2001). Si les bienfaits du sentiment d'efficacité personnelle des enseignants sur leur pratique et sur l'influence positive qu'elle a sur leurs élèves ne sont plus à prouver, il reste malgré tout de nombreuses questions ouvertes concernant la manière de le mesurer (Duffin et al., 2012; Henson, 2001). D'où l'intérêt de mettre sur pied un outil mesurant le sentiment d'efficacité personnelle des enseignants du secondaire I concernant l'un des plus grands défis du métier et un aspect particulièrement important à cette étape de la scolarité des élèves: la gestion des comportements en classe. Le fait de s'intéresser à une facette de la gestion de classe plutôt qu'à son intégralité permet une sensibilité plus élevée de l'outil et une approche plus contextualisée.

\section{Méthodologie}

Le sentiment d'efficacité personnelle ne pouvant être considéré comme un trait global d'une personne, Bandura (2006) recommande de créer des échelles adaptées en fonction de domaines précis. Notre échelle a été élaborée en fonction 
de ses recommandations en trois étapes principales: l'élaboration des items, suivie d'un pré-test et d'un test.

\section{Validité de contenu}

Pour créer une échelle mesurant le sentiment d'efficacité personnelle, Bandura (2006) recommande de commencer par une analyse conceptuelle de l'objet de mesure. Dans ce cas, il s'agit de la gestion des comportements. Une évaluation complète du sentiment d'efficacité personnelle est liée aux facteurs comportementaux sur lesquels une personne peut exercer un certain contrôle (Bandura, 2006). C'est pourquoi le cadre conceptuel de cette recherche est axé principalement sur le comportement de l'enseignant et non sur celui des élèves. L'étude de ces comportements a servi de base pour l'élaboration des items.

\section{Formulation des items et de la consigne}

Afin de s'assurer que l'échelle mesure bien un sentiment lié à l'aptitude et ne contienne pas des éléments liés à l'intention, l'estime de soi ou encore au locus de contrôle, chaque item utilise l'expression "Je suis capable de ...» (Bandura, 2006). De plus, il est spécifié que l'enseignant doit se sentir capable de faire cela de manière régulière et répétée, tout au long de l'année et non de manière ponctuelle, cela dans le but de vérifier l'efficacité personnelle autorégulatrice: c'est la capacité de renouveler un comportement aussi régulièrement que cela est nécessaire ou souhaitable (Bandura, 2006), un aspect particulièrement important en ce qui concerne la gestion des comportements.

\section{Représentativité des items}

Afin d'assurer la représentativité des contenus de l'échelle, chacun des comportements que peut adopter un enseignant a été répertorié en fonction des quatre phases (prévention, soutien, correction et remédiation) et des trois approches (interventionniste, interactionniste et non-interventionniste) en gestion des comportements. Chaque comportement répertorié a constitué un item (annexe I). Il existe plusieurs types de comportements pour chacune des phases et approches favorisées, s'inscrivant dans l'une des quatre dimensions précédemment expliquées. 50 items sont choisis selon leur pertinence, en s'assurant qu'en adoptant un tel comportement, l'enseignant ne va pas nuire à l'intégration sociale de l'élève, à son implication, à son sentiment d'appartenance ou à son identité.

\section{Accord inter-juges}

Sur la base d'un accord interjuges $(\mathrm{N}=5 ; 1$ formateur d'enseignants spécialisé en gestion de classe, 3 enseignants du secondaire I et un directeur d'établissement), 7 items ont été exclus du questionnaire et certains items ont été reformulés à la demande de deux experts pour les rendre plus clairs et plus faciles à classer selon les quatre phases de gestion des comportements en classe. Par exemple, le mot 
«punition» des items 9 et 12 n'était pas le terme favorisé lors de la conception du questionnaire mais s'est trouvé être le terme le plus compréhensible pour les enseignants. Celui-ci fait référence à toute sanction pouvant être appliquée au sein de l'école, sans les connotations négatives qui lui sont parfois attribuées (par exemple: menaces, humiliations, etc.). Il reste 43 items dans le questionnaire à la suite de cette étape.

\section{outils}

\section{L'échelle de mesure du sentiment d'efficacité personnelle des enseignants du secondaire I}

C'est une grille à jugement unique. Les sujets indiquent leur sentiment d'être capable ou non de mener à bien une situation à l'aide d'une échelle de Likert allant de 0 à 7 ( $0=$ Je ne suis pas capable du tout; 7 = Je suis tout à fait capable). Pour la phase pilote, une échelle de 43 items a été utilisée. Pour la rendre plus accessible aux enseignants, une version on-line a été faite. Avant de commencer à répondre aux questions, les enseignants ont été informés de qui menait la recherche, des buts principaux de celle-ci et du temps approximatif pour répondre à l'entier du questionnaire. De plus, il a été explicité que leur participation est volontaire et leur anonymat garantit. Enfin, il leur a été précisé qu'il n'y a pas de réponses justes ou fausses mais qu'il est important qu'elles correspondent à leur ressenti.

La seconde récolte de données s'est faite à l'aide de la version courte de l'échelle de 16 items, adaptée grâce aux informations récoltées dans le cadre de la phase pilote. Elle s'est déroulée sous les mêmes conditions que cette dernière.

\section{Questionnaire démographique}

Ce questionnaire a permis de récolter des informations complémentaires dont le sexe et l'âge.

\section{Échantillon}

\section{Échantillon, pré-test}

185 enseignants titulaires d'une maîtrise de classe du secondaire I, issus de six cycles d'orientation du canton de Fribourg en Suisse ont été sollicités à l'aide d'un questionnaire en ligne regroupant toutes les questions issues des différents questionnaires. 98 d'entre eux ont répondu à l'entier du questionnaire. Ils ont répondu aux questions en fonction de leur classe principale. Parmi cet échantillon de 98 enseignants se trouvent 49 femmes et 49 hommes âgés de 26 à 60 ans, pour un âge moyen de 41.1 ans avec un écart-type de 8.6.

\section{Échantillon, test}

245 enseignants du secondaire I issus de trois cycles d'orientation du canton de Fribourg en Suisse ont été sollicités. Ils ont également été invités à répondre à un questionnaire en ligne regroupant plusieurs questionnaires. 99 d'entre eux ont répondu à l'entier du questionnaire sur le sentiment d'efficacité personnelle. 
Parmi cet échantillon de 99 enseignants se trouvent 49 femmes et 32 hommes (18 données manquantes) âgés de 23 à 64 ans, pour un âge moyen de 41.5 ans avec un écart-type de 10.7 (19 données manquantes).

\section{Analyse des items - pré-test}

Une fois l'échelle construite, Bandura (2006) recommande de pré-tester les items, supprimer ou modifier les items ambigus et éliminer les items pour lesquels la majorité de l'échantillon répond la même chose: ces derniers ne différencient pas suffisamment les répondants. De plus, les items pour lesquels la majorité s'évalue comme très efficace ne présentent pas un défi suffisamment élevé pour différencier les répondants: ils sont dit trop faciles. L'échelle obtenue à l'issue du pré-test n'est donc pas un récapitulatif de tout ce qu'un enseignant est censé pouvoir faire pour bien gérer les comportements au sein de sa classe mais une sélection d'items permettant de différencier les enseignants en fonction de leur sentiment d'efficacité personnelle. Les échelles de mesure du sentiment d'efficacité personnelle sont souvent utilisées en même temps que d'autres questionnaires (Gaudreau et al., 2015). De plus, celle-ci étant destinée à être associée à d'autres outils de formation, il est intéressant de proposer une version plus courte de l'échelle. Le modèle proposé est pré-testé à l'aide d'une analyse en composantes principales (ACP) avec rotation orthogonale Varimax menée avec le logiciel SPSS. Avant de conduire une analyse en composantes principales, il convient de mesurer l'adéquation de l'échantillonnage à l'aide de l'indice de Kaiser-Meyer-Olkin (KMO) pour savoir si les corrélations entre items sont suffisantes $(\mathrm{KMO}>0.70)$ pour justifier ce type d'analyse (Kaiser, 1974). Ensuite, il est également recommandé de conduire un test de sphéricité de Bartlett permettant de vérifier si la matrice de corrélation est une matrice identité. Pour ce test, il est espéré que le test soit significatif $(\mathrm{p}<0,05)$ (Snedecor \& Cochran, 1989). Enfin, la fiabilité est mesurée à l'aide de l'alpha de Cronbach. La valeur est considérée comme satisfaisante dès .70 (Cortina, 1993).

\section{Analyse des items - Phase de test}

Avant la phase test, plusieurs items ont été reformulés afin de rendre les situations présentées plus difficiles et éviter ainsi les effets plafond. Par exemple, l'item "d'inclure les parents dans la résolution de problèmes de discipline» devient «d'inclure tous les parents dans la résolution de problèmes de discipline, y compris les moins coopératifs».

Bandura (2006) recommande de vérifier la validité à l'aide d'une analyse factorielle. Une analyse factorielle confirmatoire est donc menée avec le logiciel AMOS. Campbell et Fiske (1959) proposent deux aspects pour évaluer la validité de construit d'un test: la validité convergente et la validité discriminante. La validité convergente vérifie que tous les éléments d'un modèle de mesure sont statistiquement significatifs. L'indicateur retenu est: l'Average Variance Extracted, $(A V E)>0.5$ (Fornell \& Larcker, 1981). La fiabilité étant également 
un indice de validité convergente, l'alpha de Cronbach est vérifié. La valeur est considérée comme satisfaisante dès .70 (Cortina, 1993).

La validité divergente vérifie que les variables latentes sont distinctes les une des autres, ce test est particulièrement indiqué lorsqu'il existe des intercorrélations de construction élevées. Les indicateurs retenus sont dans un premier temps la comparaison de l'Average Variance Extracted (AVE) au Maximum Shared Variance $(M S V)$ et la comparaison de l'AVE à l'Average Shared Variance (ASV): $M S V<A V E$; ASV < AVE. Le dernier indicateur retenu est la comparaison de la racine carrée de l'AVE d'une variable à son coefficient de corrélation avec les autres variables. La racine carrée de l'AVE doit être supérieur aux coefficients de corrélation (Fornell \& Larcker, 1981).

\section{Résultats}

\section{Résultats du pré-test}

\section{Retrait des items présentant des effets plafond}

Pour commencer, 10 items apparaissant avec un effet plafond dans les résultats ont été retirés de l'échelle qui contenait 43 items. Ceux-ci ont été choisis car la majorité de l'échantillon a coché le 7 avec des valeurs d'asymétrie variant de -4.27 à -1.82 , signifiant qu'ils ne différencient pas suffisamment les répondants du fait qu'ils ne présentent pas une difficulté ou un défi suffisant pour une partie des enseignants (Bandura, 2006).

\section{Indice KMO et test de sphéricité de Bartlett}

L'indice KMO est de 0.87 et peut être considéré comme méritoire (Kaiser, 1974). Les corrélations entre les items sont donc suffisantes pour justifier ce type d'analyse. Le test de sphéricité de Bartlett est significatif $(\mathrm{p}=0.00)$. L'hypothèse nulle voulant qu'il s'agisse d'une matrice «identité» peut être rejetée.

\section{$A C P$}

L'ACP menée sans indication en rapport avec le nombre de facteurs à extraire n’a pas donné de résultats pouvant être théoriquement expliqués. Le nombre de facteurs à extraire a de ce fait été fixé à quatre pour correspondre au modèle théorique. De plus, l'échelle a été réduite de manière à éliminer les items ambigus: ceux-ci corrélaient avec plusieurs dimensions qui ne s'expliquaient pas théoriquement. L'élimination des items a été faite de manière à garder un bon Alpha de Cronbach, assurant ainsi la fiabilité de la mesure.

A l'issu de l'ACP, l'item 10 pressenti comme appartenant à la dimension Implication des parents est déplacé dans la dimension Gestion proactive. Malgré le fait que celui-ci fasse référence à la communication avec les parents, il semblerait que l'aspect proactif de l'item, à savoir l'encouragement des bons comportements comme le signalement de comportements déviants prenne le dessus. Cet 
item ainsi que certains autres corrèlent avec deux dimensions. Toutefois, ces corrélations peuvent être théoriquement expliquées. Il a donc été choisi de les garder pour la raison qu'ils différencient bien les enseignants les un par rapport aux autres en fonction de leur sentiment d'efficacité personnelle. Il reste 16 items à l'issu de cette étape.

Tableau 1: Saturations factorielles pour l'analyse en composantes principales

\begin{tabular}{|c|c|c|c|c|}
\hline & Gestion proactive & Gestion réactive & $\begin{array}{l}\text { Implication } \\
\text { de personnes } \\
\text { externes }\end{array}$ & $\begin{array}{l}\text { Implication des } \\
\text { parents }\end{array}$ \\
\hline ITEM 15 & .85 & .06 & .18 & .15 \\
\hline ITEM 11 & .82 & .09 & .15 & .26 \\
\hline ITEM 6 & .71 & .42 & -.06 & .17 \\
\hline ITEM 3 & .64 & .28 & .21 & .25 \\
\hline ITEM 2 & .63 & .44 & .16 & .16 \\
\hline ITEM 10 & .62 & .16 & .23 & .49 \\
\hline ITEM 12 & .07 & .81 & .21 & .16 \\
\hline ITEM 8 & .35 & .77 & .24 & .08 \\
\hline ITEM 9 & .10 & .70 & .50 & .05 \\
\hline ITEM 5 & .36 & .63 & .01 & .37 \\
\hline ITEM 13 & .54 & .60 & .10 & -.06 \\
\hline ITEM 14 & .14 & .22 & .86 & .10 \\
\hline ITEM 7 & .19 & .26 & .82 & .13 \\
\hline ITEM 1 & .20 & .05 & .26 & .82 \\
\hline ITEM 4 & .33 & .34 & -.15 & .68 \\
\hline ITEM 16 & .23 & .04 & .57 & .62 \\
\hline$\%$ de la variance & 46.56 & 10.95 & 9.55 & 5.80 \\
\hline
\end{tabular}

\section{Fiabilité}

Tableau 2: Données descriptives et consistance interne de la version courte de 16 items et de ses 4 dimensions

\begin{tabular}{lccccc}
\hline & $\begin{array}{c}\text { a de } \\
\text { Cronbach }\end{array}$ & M & SD & A & K \\
\hline Echelle de 16 items & .92 & 5.70 & 0.80 & -1.60 & 6.45 \\
\hline Gestion proactive & .90 & 5.51 & 0.94 & -1.26 & 3.44 \\
\hline Gestion réactive & .86 & 6.12 & 0.74 & -2.90 & 15.88 \\
\hline Implication des parents & .74 & 5.22 & 1.22 & -0.52 & -0.51 \\
\hline Implication de personnes externes & .87 & 5.99 & 1.18 & -1.67 & 3.29 \\
\hline
\end{tabular}


Les items de l'échelle et des différentes dimensions sont homogènes, l'échelle est fiable.

\section{Résultats - test}

Analyse factorielle confirmatoire

Tableau 3: Données descriptives et validité convergente

\begin{tabular}{lcccccc}
\hline & AVE & $\begin{array}{c}\text { a de } \\
\text { Cronbach }\end{array}$ & M & SD & A & K \\
\hline Echelle de 16 items & - & .89 & 5.48 & 0.78 & -0.55 & 0.93 \\
\hline Gestion proactive & 0.42 & .81 & 5.35 & 0.89 & -0.84 & 1.49 \\
\hline Gestion réactive & 0.44 & .78 & 6.01 & 0.74 & -0.77 & 0.60 \\
\hline Implication des parents & 0.72 & .73 & 4.77 & 1.29 & -0.60 & 0.13 \\
\hline Implication de personnes externes & 0.50 & .82 & 5.65 & 1.25 & -1.14 & 1.14 \\
\hline
\end{tabular}

L'AVE pour les dimensions: Implication des parents et Implication de personnes externes est respectivement très bon et acceptable. Toutefois, l'AVE pour les dimensions Gestion proactive et Gestion réactive se trouvent juste en dessous du seuil d'acceptabilité.

Les alphas de Cronbach obtenus pour chacune des dimensions et pour l'échelle étant supérieur à 0.7 indiquent que les items sont très homogènes.

\section{Tableau 4: Validité divergente}

\begin{tabular}{lccccccc}
\hline Variables & AVE & MSV & ASV & $\begin{array}{c}\text { Gestion } \\
\text { proactive }\end{array}$ & $\begin{array}{c}\text { Gestion } \\
\text { réactive }\end{array}$ & $\begin{array}{c}\text { Impli- } \\
\text { cation } \\
\text { parents }\end{array}$ & $\begin{array}{c}\text { Impli- } \\
\text { cation } \\
\text { personnes } \\
\text { extérieures }\end{array}$ \\
\hline Gestion proactive & 0.42 & 0.65 & 0.48 & $\mathbf{0 . 2 1}$ & & & \\
\hline Gestion réactive & 0.44 & 0.44 & 0.57 & 0.66 & $\mathbf{0 . 2 2}$ & & \\
\hline Implication parents & 0.72 & 0.65 & 0.41 & 0.80 & 0.44 & $\mathbf{0 . 3 6}$ & \\
\hline $\begin{array}{l}\text { Implication } \\
\text { personnes externes }\end{array}$ & 0.50 & 0.39 & 0.34 & 0.60 & 0.52 & 0.63 & $\mathbf{0 . 2 5}$ \\
\hline
\end{tabular}

Note: la racine carrée de l'AVE est sur la diagonale de la matrice de corrélation en gras.

Concernant le premier indicateur de validité divergente, les résultats observés concernant les dimensions Implication des parents et Implication de personnes sont positifs: AVE > MSV. Le résultat relevé de la dimension Gestion réactive est juste en dessous de la limite acceptable: $\mathrm{AVE}=\mathrm{MSV}$. Le résultat observé pour la dimension Gestion proactive en revanche, est insuffisant: AVE < MSV. Les résultats du second indicateur sont similaires: les dimensions Implication des parents et Implication de personnes externes obtiennent de bons résultats, ASV < 
AVE. Toutefois, les dimensions Gestion proactive et Gestion réactive obtiennent des résultats insuffisants, ASV > AVE. Enfin, la racine carrée de l'AVE est inférieure aux coefficients de corrélation, pour tous les construits du modèle. Par conséquent, celui-ci manifeste une faible validité divergente.

\section{Discussion et conclusion}

La présente étude explique la construction et les premiers éléments de validation de l'échelle de sentiment d'efficacité personnelle en gestion des comportements en classe spécifique au secondaire I, basée sur les recommandations de Bandura (2006). Cette approche permet de vérifier la validité de contenu dans un premier temps: cette validité a été vérifiée à partir d'une analyse conceptuelle approfondie. Les items sont ensuite testés à l'aide d'une phase de pré-test durant laquelle une analyse en composantes principales est conduite. La validité de construit est vérifiée à l'aide d'une phase de test durant laquelle une analyse factorielle confirmatoire est faite. Enfin, la fiabilité de l'échelle est mesurée lors de la phase de pré-test ainsi que lors de la phase test.

Les résultats obtenus lors de l'analyse en composantes principales montrent que plusieurs items corrèlent avec plusieurs dimensions. Malgré une reformulation de certains items avant la phase de test et l'analyse factorielle confirmatoire, les résultats obtenus ne sont pas ceux espérés. En effet, les dimensions ne se distinguent pas suffisamment les unes des autres. Ainsi, nous sommes amenés à nous demander si les corrélations entre dimensions sont dues à un problème lié au modèle ou au fait que les différentes dimensions font appel à des compétences sous-jacentes similaires, comme cela est possible lors de co-variation entre différents domaines ou dimensions (Bandura, 2006). L'échelle étant élaborée à partir du contenu de différents modèles reconnus de longue date (Charles, 2009; Glickman \& Tamashiro, 1980; Legault, 2001) en ce qui concerne la gestion des comportements et prenant en compte un modèle plus récent s'appuyant sur de nombreuses observations (Sieber, 2000), la validité de contenu de l'échelle n'est pas remise en question. De plus, les items restant dans la version courte sont ceux qui permettent le mieux de différencier les enseignants en fonction de leur sentiment d'efficacité. Toutefois, il serait intéressant de mener l'étude du modèle de gestion des comportements plus loin. Il serait intéressant par exemple de le faire par un autre moyen tel que des observations ou par l'utilisation d'une échelle mesurant des intentions plutôt que le sentiment d'efficacité personnelle. Cela permettrait de peut-être mieux différencier les dimensions de la gestion des comportements et ainsi de mieux comprendre son fonctionnement, ce qui permettrait ensuite de confirmer ou non l'hypothèse que l'échelle présentée est en fait unidimensionnelle comme le laissent penser les résultats observés plutôt que divisée en quatre dimensions. 
Il reste donc à clarifier certaines ambiguïtés au niveau de la validité de construit de l'échelle présentée. Il n'en demeure pas moins que cet outil est fiable si l'on se base sur les alphas de Cronbach obtenus qui sont très bons. Sachant que les échelles spécifiques et contextualisées sont de meilleurs prédicteurs de comportement que des échelles plus générales (Pajares, 1996), cette échelle reste malgré tout un outil intéressant à inclure dans la formation, l'évaluation formative, l'auto-évaluation ou encore la recherche en lien avec les pratiques enseignantes en ce qui concerne la gestion des comportements au secondaire I.

Cette étude présente certaines limites, à commencer par son faible échantillon. Il serait intéressant de refaire la même étude auprès d'un échantillon plus grand afin de vérifier les résultats obtenus. De plus, la distribution des scores sur les différents items présente des effets plafond. Il serait donc intéressant d'envisager une échelle de Likert plus grande. Enfin, de premiers éléments de la validité ont été abordés mais il serait intéressant d'aller plus loin en s'intéressant à la validité critérielle par exemple, et ce d'autant plus qu'il s'agit d'une échelle de mesure du sentiment d'efficacité personnelle qui est un sentiment reconnu comme étant un bon prédicteur de comportements (Bandura, 2006).

\section{Notes}

1 Pour des raisons de commodité de lecture, nous avons renoncé à féminiser les catégories de personnes et de fonctions. Nous remercions nos lectrices et nos lecteurs de leur compréhension.

2 Le secondaire I fait référence aux degrés $9 \mathrm{H}, 10 \mathrm{H}$ et $11 \mathrm{H}$ du système scolaire suisse romand. Les élèves sont en principe âgés de 12 à 16 ans. Il s'agit des trois dernières années de scolarité obligatoire.

\section{Références}

Abu-Tineh, A. M., Khasawneh, S. A. \& Khalaileh, H. A. (2011). Teacher self-efficacy and classroom management styles in Jordanian schools. Management in Education. 25, (4), 175-181.

Adams, K. S. \& Christenson, S. L. (2000). Trust and the family-school relationship, examination of parent-teacher differences in elementary and secondary grades. Journal of school psychology, 38, (5), 477-497.

Bandura, A. (1977). Self-efficacy: Toward a unifying theory of behavioral change. Psychological Review, 84, (2), 191-215.

Bandura, A. (1986). Social foundations of thought and action: social cognitive theory. Englewood Cliffs, NJ: Prentice-Hall.

Bandura, A. (2006). Guide for constructing self-efficacy scales. In F. Pajares \& T. Urban (Éd.), Self-efficacy beliefs of adolescents (pp. 307-337). Greenwich, CT: Information Age.

Bandura, A. (2013). Auto-efficacité: le sentiment d'efficacité personnelle. Bruxelles: De Boeck.

Blaya, C., Gilles, J.-L., Plunus, G. \& Tièche, Ch. (2011). Accrochage scolaire et alliances éducatives: vers une intégration des approches scolaires et communautaires. Education et francophonie, 39, (2), 227-249.

Brown, A. L., Lee, J. \& Collins, J. (2015). Does student teaching matter? Investigating pre-service teachers' sense of efficacy and preparedness. Teaching Education, 26, (1), 77-93.

Campbell, D. T. \& Fiske, D. W. (1959). Convergent and discriminant validation by the multitrait-multimethod matrix. Psychological bulletin, 56-81. 
Canter, L. (2010). Assertive discipline: Positive behavior management for today's classroom. Bloomington, IN: Solution tree press.

Charles, C. M. (2009). La discipline en classe: modèles, doctrines et conduites. Bruxelles: De Boeck Université.

Christophersen, K. A., Elstad, E., Turmo, A. \& Solhaug, T. (2016). Teacher education programmes and their contribution to student teacher efficacy in classroom management and pupil engagement. Scandinavian journal of educational research, 60, (2), 240-254.

Cortina, J. M. (1993). What is coefficient alpha? An examination of theory and applications. Journal of applied psychology, 78, (1), 98-104.

Deemer, S. A. \& Minke, K. M. (1999). An investigation of the factor structure of the teacher efficacy scale. The journal of educational research, 93, (1), 3-10.

Dembo, M. H. \& Gibson, S. (1985). Teachers' sense of efficacy: An important factor in school improvement. The elementary school journal, 86, (2), 173-184.

De Saedeleer, S., Brassard, A. \& Brunet, L. (2004). Des écoles plus ouvertes à l'implication des parents? Le point de vue des directeurs d'établissement au Québec. Revue Française de Pédagogie, 147, 69-77.

Dicke, T., Elling, J., Schmeck, A. \& Leutner, D. (2015). Reducing reality shock: The effects of classroom management skills training on beginning teachers. Teaching and teacher education, 48, 1-12.

Doyle, W. (2006). Ecological approaches to classroom management. In C. Evertson \& C. Weinstein (Éd.), Handbook of Classroom Management: Research, practice and contemporary issue (pp. 97-125). London: Lawrence Erlbaum Associates.

Dreikurs, R. (1968). Psychology in the classroom: a manual for teachers (2ème éd.). New York, NY: Harper \& Row.

Duffin, L. C., French, B. F. \& Patrick, H. (2012). The teachers' sense of efficacy scale: Confirming the factor structure with beginning pre-service teachers. Teaching and teacher education, 28, 827-834.

Eisenman, G., Edwards, S. \& Cushman, C. A. (2015). Bringing Reality to Classroom Management in Teacher Education. The professional educator, 39, (1), 1-12.

Erikson, E. H. (1972). Adolescence et crise. Paris: Flammarion.

Fornell, C. \& Larcker, D. F. (1981). Evaluating structural equation models with unobservable variables and measurement error. Journal of marketing research, 39-50.

Freud, A. (1978). Le moi et les mécanismes de défense. (9ème éd., version française). Paris: PUF.

Gaudreau, N., Frenette, E. \& Thibodeau, S. (2015). Elaboration de l'échelle du sentiment d'efficacité personnelle des enseignants en gestion de classe (ESEPGC). Mesure et évaluation en éducation, 38, (2), 31-60.

Gaudreau, N., Royer, E., Beaumont, C. \& Frenette, E. (2012). Le sentiment d'efficacité personnelle des enseignants et leurs pratiques de gestion de la classe et des comportements difficiles des élèves. Canadian journal of education / Revue canadienne de l'éducation, 35, (1), 82-101.

Glickman, C. \& Tamashiro, R. (1980). Clarifying teachers' beliefs about discipline. Educational Leadership, 37, (6), 459-464.

Gonzalez-DeHass, A. R., Willems, P. P. \& Doan Holbein, M. F. (2005). Examining the relationship between parental involvement and student motivation. Educational psychology review, 17, (2), 99-123.

Gordon, T. (2009). Eduquer sans punir: apprendre l'autodiscipline aux enfants. Paris: Marabout.

Hamilton, L. (2015). Early professional development in the Scottish context: pre-service high school teachers and the management of behavior in classrooms. Teacher development, 19, (3), 328-343.

Henson, R. K. (2001). Teacher self-efficacy: Substantive implications and measurement dilemmas. Paper presented at the annual meeting of the educational research exchange. Consulté le 
10 avril 2017 dans https://www.researchgate.net/publication/2365774_Teacher_Self-Efficacy_Substantive_Implications_and_Measurement_Dilemmas

Hoover-Dempsey, K. V., Walker, J. M. T., Jones, K. P. \& Reed, R. P. (2002). Teachers involving parents (TIP): Results of an in-service teacher education program for enhancing parental involvement. Teaching and teacher education, 18, (7), 843-867.

Kaiser, H. (1974). An index of factor simplicity. Psychometrika, 39, 31-36.

Kounin, J. S (1970). Discipline and group management in classrooms, New York, NY: Holt, Rinehart \& Winston.

Legault, J. P. (2001). Gestion de classe et discipline: une compétence à construire. Outremont: Logiques.

Léveillé, C.-J. \& Dufour, F. (1999). Les défis de la gestion de classe au secondaire. Revue des sciences de l'éducation, 25, (3), 515-532.

Malmgren, K. W., Trezek, B. J. \& Paul, P. V. (2005). Models of classroom management as applied to the secondary classroom. Clearing House: A Journal of Educational Strategies, Issues and Ideas, 79, (1), 36-39.

Martineau, S. \& Gauthier, C. (1999). La gestion de classe au cour de l'effet enseignant. Revue des sciences de l'éducation, 25, (3), 467-496.

Nault, T. \& Fijalkow, J. (1999). Introduction. La gestion de classe: d'hier à demain. Revue des sciences de l'éducation, 25, (3), 451-466.

Pajares, F. (1996). Self-efficacy beliefs in academic settings. Review of educational research, 66, 543-578.

Piaget, J. \& Inhelder, B. (1966). La psychologie de l'enfant. Paris: PUF.

Ritchie, K. R. (2006). A comparison of the self-efficacy scores of preservice teachers based on initial college experience. Unpublished doctoral dissertation, University of North Texas.

Schwarzer, R., Schmitz, G. S. \& Daytner, G. T. (1999). Teacher self-efficacy. Consulté le 25 mai 2017 dans http://userpage.fu-berlin.de/-health/teacher_se.htm

Sempowicz, T. \& Hudson, P. (2011). Analysing mentoring dialogues for developing a preservice teacher's classroom management practices. The Australian journal of teacher education, 36, (8), 1-16.

Sieber, M. (2000). Comment gérer 1' indiscipline en classe? Gérer l'indiscipline auprès d'élèves hyperactifs, oppositionnels ou provocateurs. Fribourg: Editions universitaires Fribourg, Suisse.

Snedecor, G. W. \& Cochran, W. G. (1989). Statistical Methods. (8ème éd.). Iowa, IA: Iowa State University Press.

Tschannen-Moran, M. \& Woolfolk Hoy, A. (2001). Teacher efficacy: capturing an elusive construct. Teaching and teacher education, 17, 783-805.

Tschannen-Moran, M., Woolfolk Hoy, A. \& Hoy, W.K. (1998). Teacher efficacy: Its meaning and measure. Review of educational research, 68, (2), 202-248.

Wagner, J. A. (1987). Formal operations and ego identity in adolescence. Adolescence, 22, 23-35.

Woolfolk Hoy, A. (2004). What do teachers know about self-efficacy? Paper presented at the annual meeting of the American Educational Research Association, San Diego, CA.

Mots-clés: Gestion des comportements, gestion de classe, sentiment d'efficacité personnelle, secondaire I, formation des enseignants 


\section{Selbstwirksamkeit für das Verhaltensmanagement. Entwicklung und Validierung einer Messskala}

\section{Zusammenfassung}

Die Forschung zur Selbstwirksamkeit und deren Messung zeigt, dass es sich nicht um ein globales Merkmal handelt, sondern um eine Reihe von Überzeugungen, die ein Individuum über verschiedene Aspekte seiner Funktionsweise haben kann. Dieser Artikel stellt die Konstruktion und die ersten Elemente der Validierung einer Skala zur Messung der Selbstwirksamkeit hinsichtlich des Verhaltensmanagements in Klassen der Sekundarstufe I vor. Sie wurde nach den Empfehlungen von Bandura (2006) mit einer Gruppe von Lehrpersonen in zwei Stufen entwickelt und validiert: eine Pilotphase, gefolgt von einer Testphase. Die Validität des Inhalts wurde mittels einer gründlichen konzeptionellen Analyse sowie der Einschätzungsverlässlichkeit zwischen Personen überprüft. Die Validität des Konstrukts wurde mit einer Hauptkomponentenanalyse während der Pilotphase und anschliessend einer konfirmatorischen Faktorenanalyse während der Testphase verifiziert. Diese Analysen werfen Fragen hinsichtlich der Validierung des verwendeten Modells auf. Die endgültige Version enthält 16 Items und erfüllt die Bedingungen der Reliabilität.

Schlagworte: Verhaltensmanagement, Klassenführung, Selbstwirksamkeit, Mittelschule, Lehrerausbildung

\section{Sentimento di efficacia personale nella gestione dei comportamenti. Elaborazione e validazione di una scala di misura}

\section{Riassunto}

Gli studi sul sentimento di efficacia personale e sulla maniera di misurarlo mostrano che non si tratta di una caratteristica globale ma di un insieme di convinzioni che una persona può avere riguardo a diversi aspetti del proprio modo di agire. Il presente articolo spiega il processo di costruzione e i primi elementi di validazione di una scala di misura del sentimento di efficacia personale orientata in modo specifico alla gestione dei comportamenti nelle classi del livello secondario I. L'elaborazione e la validazione della scala di misura sono state realizzate tenendo conto dei principi esposti da Bandura (2006) e facendo capo a dei campioni di insegnanti sia per la fase pilota che per la fase di test. La validità di contenuto è stata controllata con un'analisi concettuale approfondita e un accordo tra giudici. La validità di costrutto è stata dapprima verificata con un'analisi in componenti principali effettuata durante la fase pilota ; essa è poi 
stata verificata con un'analisi fattoriale confermativa durante la fase di test. Tali analisi sollevano delle domande riguardo alla validazione del modello utilizzato. La versione finale conta 16 items e soddisfa le condizioni di attendibilità.

Parole chiave: Gestione dei comportamenti, gestione della classe, sentimento di efficacia personale, livello secondario I, formazione degli insegnanti

\section{Self-Efficacy in Behaviour Management. Development and Validation of a Measurement Scale}

\section{Summary}

Research on self-efficacy and its measurement shows that it isn't a global characteristic, but rather a set of beliefs that an individual may have about different aspects of his or her functioning. This article presents the development of the self-efficacy scale for behaviour management in secondary school classrooms as well as initial elements of validation. It was developed and validated according to Bandura's (2006) recommendations among a population of teachers throughout two stages: a pilot phase followed by a test phase. Content validity was verified through thorough conceptual analysis and inter-judicial agreement. Construct validity was verified using a principal component analysis during the pilot phase and then a confirmatory factor analysis during the test phase. These analyzes raise questions regarding the validation of the used model. The final version contains 16 items and satisfies the conditions of reliability and criterion validity.

Key words: Behaviour management, classroom management, self-efficacy, middle school, teacher training 


\section{Annexe 1}

4 phases et trois approches de la gestion des comportements au secondaire I Classifications des comportements d'enseignant

\begin{tabular}{|c|c|c|c|}
\hline \multicolumn{4}{|c|}{ PREVENTION } \\
\hline & Interventionniste & Interactionniste & Non-interventionniste \\
\hline $\begin{array}{l}\text { Attitude de } \\
\text { l'enseignant }\end{array}$ & $\begin{array}{l}\text { L'enseignant se maintient } \\
\text { de façon à affirmer son } \\
\text { statut supérieur. } \\
\text { L'enseignant établit un } \\
\text { rythme de travail. } \\
\text { L'enseignant utilise l'effet } \\
\text { de réverbération. } \\
\text { L'enseignant insiste sur } \\
\text { les bonnes manières. }\end{array}$ & $\begin{array}{l}\text { L'enseignant donne } \\
\text { l'exemple en se } \\
\text { comportant comme il } \\
\text { aimerait que ses élèves se } \\
\text { comportent. } \\
\text { L'enseignant se montre } \\
\text { juste mais ferme par } \\
\text { rapport aux règles de vie } \\
\text { établies en commun. } \\
\text { L'enseignant croit en la } \\
\text { maîtrise de soi des élèves } \\
\text { et cherche à la favoriser. }\end{array}$ & $\begin{array}{l}\text { L'enseignant incite les } \\
\text { élèves à coopérer sans les } \\
\text { forcer. } \\
\text { L'enseignant exprime ses } \\
\text { sentiments à la première } \\
\text { personne de manière } \\
\text { à prévenir certains } \\
\text { comportements. }\end{array}$ \\
\hline Statut des élèves & $\begin{array}{l}\text { L'enseignant et les } \\
\text { élèves n'ont pas des } \\
\text { statuts égalitaires : c'est } \\
\text { l'enseignant qui décide } \\
\text { et qui est en position de } \\
\text { pouvoir. }\end{array}$ & $\begin{array}{l}\text { L'enseignant cherche } \\
\text { à établir une relation } \\
\text { fondée sur le respect et la } \\
\text { confiance avec ses élèves } \\
\text { qui sont traités comme } \\
\text { des égaux sociaux. Le } \\
\text { pouvoir décisionnel reste } \\
\text { à l'enseignant. }\end{array}$ & $\begin{array}{l}\text { L'avis des élèves vaut } \\
\text { autant que celui de } \\
\text { l'enseignant, ils sont } \\
\text { parfaitement égaux. }\end{array}$ \\
\hline Le règlement & $\begin{array}{l}\text { Etablissement d'un } \\
\text { règlement et de } \\
\text { conséquences par } \\
\text { l'enseignant et l'école. }\end{array}$ & $\begin{array}{l}\text { L'élève est responsabilisé, } \\
\text { il participe aux décisions } \\
\text { et à l'élaboration du } \\
\text { règlement. } \\
\text { L'enseignant fait } \\
\text { connaître le règlement } \\
\text { établi en commun au } \\
\text { directeur. }\end{array}$ & $\begin{array}{l}\text { Les règles de classe et les } \\
\text { décisions sont faites de } \\
\text { manière démocratique. }\end{array}$ \\
\hline $\begin{array}{l}\text { Les consé- } \\
\text { quences en cas } \\
\text { d'infraction au } \\
\text { règlement }\end{array}$ & $\begin{array}{l}\text { L'enseignant informe } \\
\text { l'élève des conséquences } \\
\text { en cas d'écart de } \\
\text { conduite. } \\
\text { L'enseignant crée une } \\
\text { hiérarchie de consé- } \\
\text { quences. }\end{array}$ & $\begin{array}{l}\text { L'enseignant et les élèves } \\
\text { créent une hiérarchie } \\
\text { de conséquences que } \\
\text { l'enseignant respectera en } \\
\text { cas d'inconduite de la par } \\
\text { d'un élève. }\end{array}$ & $\begin{array}{l}\text { Aucune conséquence } \\
\text { n'est prévue à l'avance, } \\
\text { car celles-ci sont généra- } \\
\text { lement évitées. }\end{array}$ \\
\hline Les parents & $\begin{array}{l}\text { Les parents sont informés } \\
\text { du règlement de classe et } \\
\text { des conséquences en cas } \\
\text { d'inconduite. }\end{array}$ & $\begin{array}{l}\text { Les parents sont informés } \\
\text { du règlement établi par } \\
\text { l'enseignant et les élèves. }\end{array}$ & $\begin{array}{l}\text { Les parents ne sont pas } \\
\text { informés du règlement. }\end{array}$ \\
\hline
\end{tabular}


4 phases et trois approches de la gestion des comportements au secondaire I Classifications des comportements d'enseignant

\begin{tabular}{|c|c|c|c|}
\hline \multicolumn{4}{|c|}{ SOUTIEN } \\
\hline & Interventionniste & Interactionniste & Non-interventionniste \\
\hline $\begin{array}{l}\text { Attitude de } \\
\text { l'enseignant }\end{array}$ & $\begin{array}{l}\text { L'enseignant utilise } \\
\text { le langage non verbal } \\
\text { (regard direct, proximité } \\
\text { physique, expression } \\
\text { du visage, gestes) pour } \\
\text { montrer sa présence et sa } \\
\text { vigilance. } \\
\text { L'enseignant évalue le } \\
\text { système de discipline et } \\
\text { le modifie si besoin. }\end{array}$ & $\begin{array}{l}\text { L'enseignant encourage } \\
\text { ses élèves. } \\
\text { L'enseignant évalue le } \\
\text { système de discipline et } \\
\text { le modifie si besoin, en } \\
\text { collaboration avec les } \\
\text { élèves. }\end{array}$ & $\begin{array}{l}\text { L'enseignant fait } \\
\text { attention aux sentiments } \\
\text { des élèves. }\end{array}$ \\
\hline $\begin{array}{l}\text { Stratégies pour } \\
\text { favoriser le bon } \\
\text { comportement }\end{array}$ & $\begin{array}{l}\text { L'enseignant récompense } \\
\text { les bons comportements. }\end{array}$ & $\begin{array}{l}\text { L'enseignant enseigne le } \\
\text { programme de discipline } \\
\text { et le bon comportement } \\
\text { aux élèves. }\end{array}$ & $\begin{array}{l}\text { L'enseignant permet et } \\
\text { incite l'élève à commu- } \\
\text { niquer. }\end{array}$ \\
\hline Les parents & $\begin{array}{l}\text { L'enseignant tient } \\
\text { les parents informés } \\
\text { du comportement de } \\
\text { leur enfant par des } \\
\text { annotations dans le } \\
\text { carnet devoir, un coup } \\
\text { de téléphone ou une } \\
\text { rencontre. }\end{array}$ & $\begin{array}{l}\text { L'enseignant s'entretient } \\
\text { de temps en temps avec } \\
\text { les parents au sujet du } \\
\text { comportement de leur } \\
\text { enfant. }\end{array}$ & $\begin{array}{l}\text { L'enseignant ne contacte } \\
\text { pas les parents au sujet } \\
\text { du comportement de leur } \\
\text { enfant. }\end{array}$ \\
\hline
\end{tabular}


4 phases et trois approches de la gestion des comportements au secondaire I Classifications des comportements d'enseignant

\begin{tabular}{|c|c|c|c|}
\hline \multicolumn{4}{|c|}{ CORRECTION } \\
\hline & Interventionniste & Interactionniste & Non-interventionniste \\
\hline $\begin{array}{l}\text { Stratégies } \\
\text { mises en place } \\
\text { lorsqu'un } \\
\text { élève fait } \\
\text { preuve } \\
\text { d'inconduite }\end{array}$ & $\begin{array}{l}\text { L'enseignant applique une } \\
\text { conséquence. } \\
\text { L'enseignant applique } \\
\text { une conséquence selon le } \\
\text { règlement de classe ou de } \\
\text { l'école. } \\
\text { L'enseignant applique une } \\
\text { conséquence en fonction } \\
\text { de la hiérarchie préétablie. }\end{array}$ & $\begin{array}{l}\text { L'enseignant applique les } \\
\text { règles et les conséquences } \\
\text { auxquelles les élèves ont } \\
\text { contribué. }\end{array}$ & $\begin{array}{l}\text { L'enseignant exprime } \\
\text { ses sentiments face au } \\
\text { problème en utilisant la } \\
\text { première personne. }\end{array}$ \\
\hline $\begin{array}{l}\text { Stratégies } \\
\text { pour limiter } \\
\text { les compor- } \\
\text { tements } \\
\text { malvenus }\end{array}$ & $\begin{array}{l}\text { L'enseignant fait preuve } \\
\text { de vigilance en identifiant } \\
\text { les élèves fautifs, en leur } \\
\text { signalant que leur compor- } \\
\text { tement est inacceptable, } \\
\text { en nommant certains } \\
\text { élèves, en s'occupant de la } \\
\text { déviance la plus grave en } \\
\text { premier de manière rapide } \\
\text { et au bon moment. } \\
\text { L'enseignant intimide ses } \\
\text { élèves. }\end{array}$ & $\begin{array}{l}\text { L'enseignant cherche à } \\
\text { réorienter les comporte- } \\
\text { ments malvenus. } \\
\text { L'enseignant refuse toute } \\
\text { excuse visant à expliquer } \\
\text { un mauvais compor- } \\
\text { tement, fait preuve de } \\
\text { persévérance et refuse les } \\
\text { rapports de force. } \\
\text { L'enseignant discute en } \\
\text { tête à tête avec ses élèves } \\
\text { lorsque cela s'avère néces- } \\
\text { saire. }\end{array}$ & $\begin{array}{l}\text { L'enseignant modifie } \\
\text { l'environnement pour } \\
\text { faire disparaître un } \\
\text { mauvais compor- } \\
\text { tement. }\end{array}$ \\
\hline Les parents & $\begin{array}{l}\text { L'enseignant informe les } \\
\text { parents de l'inconduite de } \\
\text { leur enfant. }\end{array}$ & $\begin{array}{l}\text { L'enseignant discute avec } \\
\text { les parents de l'inconduite } \\
\text { de leur enfant. }\end{array}$ & $\begin{array}{l}\text { L'enseignant n'informe } \\
\text { pas les parents de } \\
\text { l'inconduite de leur } \\
\text { enfant. }\end{array}$ \\
\hline
\end{tabular}


4 phases et trois approches de la gestion des comportements au secondaire I Classifications des comportements d'enseignant

\begin{tabular}{|c|c|c|c|}
\hline \multicolumn{4}{|c|}{ REMEDIATION } \\
\hline & Interventionniste & Interactionniste & Non-interventionniste \\
\hline $\begin{array}{l}\text { Stratégies } \\
\text { mises en } \\
\text { place pour } \\
\text { éviter qu'un } \\
\text { comportement } \\
\text { malvenu se } \\
\text { reproduise }\end{array}$ & $\begin{array}{l}\text { Après avoir appliqué une } \\
\text { conséquence, l'enseignant } \\
\text { recrée un climat sain. } \\
\text { L'enseignant crée des } \\
\text { solutions au problème. } \\
\text { L'enseignant fait appel à } \\
\text { des personnes extérieures } \\
\text { de la classe (direction, } \\
\text { psychologue) pour } \\
\text { s'occuper de l'élève. }\end{array}$ & $\begin{array}{l}\text { L'enseignant établit un } \\
\text { dialogue avec l'élève pour } \\
\text { réfléchir au problème et } \\
\text { trouver une solution. } \\
\text { L'enseignant fait appel à } \\
\text { des personnes extérieures } \\
\text { de la classe (direction, } \\
\text { médiateur, psychologue) } \\
\text { pour l'aider à mieux } \\
\text { communiquer avec l'élève } \\
\text { en question et trouver des } \\
\text { solutions. }\end{array}$ & $\begin{array}{l}\text { L'enseignant cherche } \\
\text { à aider l'élève sans le } \\
\text { forcer, sans qu'il y ait } \\
\text { de perdant à la suite de } \\
\text { la situation probléma- } \\
\text { tique. }\end{array}$ \\
\hline $\begin{array}{l}\text { Stratégies } \\
\text { mises en place } \\
\text { lorsqu'un } \\
\text { élève faisant } \\
\text { preuve } \\
\text { d'inconduite } \\
\text { récidive une } \\
\text { ou plusieurs } \\
\text { fois }\end{array}$ & $\begin{array}{l}\text { L'enseignant applique une } \\
\text { conséquence en fonction } \\
\text { de la hiérarchie préétablie. } \\
\text { L'enseignant élabore un } \\
\text { programme individualisé } \\
\text { de comportement pour } \\
\text { un élève difficile dont les } \\
\text { conséquences differeront } \\
\text { de celles prévues pour le } \\
\text { reste de la classe. }\end{array}$ & $\begin{array}{l}\text { L'enseignant discute avec } \\
\text { l'élève difficile et élabore } \\
\text { avec lui un programme } \\
\text { individualisé de compor- } \\
\text { tement dont les consé- } \\
\text { quences differeront de } \\
\text { celles prévues pour le reste } \\
\text { de la classe. }\end{array}$ & \\
\hline Les parents & $\begin{array}{l}\text { L'enseignant crée une } \\
\text { solution au problème sans } \\
\text { l'aide des parents. Il les } \\
\text { informe de la solution. }\end{array}$ & $\begin{array}{l}\text { L'enseignant, les parents et } \\
\text { parfois l'enfant cherchent } \\
\text { une solution au problème } \\
\text { et de nouvelles possibilités. }\end{array}$ & $\begin{array}{l}\text { Les parents ne sont } \\
\text { pas inclus dans la } \\
\text { remédiation. }\end{array}$ \\
\hline
\end{tabular}

Annexe 2 (page suivante)

Echelle de sentiment d'efficacité personnelle en gestion des comportements en classe des enseignants du secondaire I

Un certain nombre de situations concernant la gestion de comportements en classe sont décrites ci-dessous. Evaluez votre certitude de pouvoir gérer chacune de ces situations de manière optimale, afin d'assurer ou de retrouver rapidement un climat sain dans la classe, ainsi qu'avec chacun des élèves.

Evaluez votre degré de certitude en cochant la case correspondante : $0=$ Je ne suis pas du tout capable $7=$ Je suis tout à fait capable. 
ATTENTION: Nous ne voulons pas savoir si vous êtes certain-e de pouvoir gérer ces situations si elle surviennent une fois, mais de manière régulière et répétée, tout au long de l'année.

\begin{tabular}{|c|c|c|c|c|c|c|c|c|c|}
\hline & $\begin{aligned} O= & \text { Je ne suis pas du tout capable } \\
& \quad=\text { Je suis tout à fait capable. }\end{aligned}$ & 0 & 1 & 2 & 3 & 4 & 5 & 6 & 7 \\
\hline 1 & $\begin{array}{l}\text { d'inclure tous les parents dans la résolution de problèmes de } \\
\text { discipline, y compris les moins coopératifs. }\end{array}$ & & & & & & & & \\
\hline 2 & $\begin{array}{l}\text { d'enseigner le programme de discipline et le bon compor- } \\
\text { tement aux élèves durant l'année scolaire. }\end{array}$ & & & & & & & & \\
\hline 3 & $\begin{array}{l}\text { de créer des stratégies efficaces, à l'avance, pour corriger le } \\
\text { comportement d'un élève difficile. }\end{array}$ & & & & & & & & \\
\hline 4 & $\begin{array}{l}\text { de communiquer aux parents mes attentes concernant la } \\
\text { discipline de leur enfant en classe dès le début de l'année } \\
\text { scolaire. }\end{array}$ & & & & & & & & \\
\hline 5 & d'intervenir aux premiers signes d'indiscipline. & & & & & & & & \\
\hline 6 & $\begin{array}{l}\text { de prendre du recul face à une situation difficile dans le but } \\
\text { de mettre en place des solutions efficaces à un problème } \\
\text { disciplinaire récurent. }\end{array}$ & & & & & & & & \\
\hline 7 & $\begin{array}{l}\text { de mettre en place une collaboration avec des personnes } \\
\text { extérieures à la classe (psychologue, médiateur, la direction) } \\
\text { pour régler un problème d'inconduite. }\end{array}$ & & & & & & & & \\
\hline 8 & $\begin{array}{l}\text { de me montrer juste mais ferme lorsqu'un élève ne respecte } \\
\text { pas les règles de classe. }\end{array}$ & & & & & & & & \\
\hline 9 & d'appliquer une punition de manière ferme. & & & & & & & & \\
\hline 10 & $\begin{array}{l}\text { de communiquer explicitement avec tous les parents au sujet } \\
\text { du comportement bon ou mauvais de leur enfant durant } \\
\text { l'année scolaire. }\end{array}$ & & & & & & & & \\
\hline 11 & $\begin{array}{l}\text { de réfléchir et trouver des solutions en coopération avec } \\
\text { l'élève difficile. }\end{array}$ & & & & & & & & \\
\hline 12 & d'appliquer une punition selon le règlement de l'école. & & & & & & & & \\
\hline 13 & $\begin{array}{l}\text { d'identifier les élèves fautifs, de leur signaler que leur } \\
\text { comportement est inacceptable. }\end{array}$ & & & & & & & & \\
\hline 14 & $\begin{array}{l}\text { de faire appel à des personnes extérieures (psychologue, } \\
\text { médiateur, direction) pour m'aider à mieux communiquer } \\
\text { avec un élève difficile afin de trouver des solutions. }\end{array}$ & & & & & & & & \\
\hline 15 & $\begin{array}{l}\text { de dialoguer de manière à ce que ni moi ni l'élève ne sorte } \\
\text { perdant à l'issu d'une situation problématique. }\end{array}$ & & & & & & & & \\
\hline 16 & $\begin{array}{l}\text { d'inclure les parents dans la recherche de nouvelles possibi- } \\
\text { lités suite à une situation difficile. }\end{array}$ & & & & & & & & \\
\hline
\end{tabular}

\section{Item key}

Gestion proactive

$2 ; 3 ; 6 ; 10 ; 11 ; 15$

Gestion réactive

5; 8; 9; 12;13

Implication parents

$1 ; 4$

Implication externe

$7 ; 14 ; 16$ 